\title{
Floating Silicon Method Single Crystal Ribbon- Observations and Proposed Limit Cycle Theory
}

\author{
Peter Kellerman ${ }^{a,}{ }^{*}$, Brian Kernan ${ }^{a}$, Brian T. Helenbrook ${ }^{b}$, Dawei Sun ${ }^{a}$, \\ Frank Sinclair ${ }^{a}$, Frederick Carlson ${ }^{b}$ \\ a Varian Semiconductor Equipment Division \\ Applied Materials Inc., Gloucester, MA, 01930, USA \\ ${ }^{b}$ Department of Mechanical and Aerospace Engineering \\ Clarkson University, Potsdam, NY, 13699-5725, USA
}

\begin{abstract}
In the Floating Silicon Method (FSM), a single-crystal Si ribbon is grown while floating on the surface of a Si melt. In this paper, we describe the phenomenology of FSM and the observation of approximately regularly spaced "facet lines" on the ribbon surface whose orientation aligns with (111) crystal planes. $\mathrm{Sb}$ demarcation experiments sectioned through the thickness of the ribbon reveal that the solid/melt interface consists of dual (111) planes and that the leading edge facet growth is saccadic in nature, rather than steady-state.
\end{abstract}

To explain this behavior, we propose a heuristic solidification limit cycle theory, using a continuum level of description with anisotropic kinetics as developed by others, and generalizing the interface kinetics to include a roughening transition as well as a re-faceting mechanism that involves curvature and the Gibbs-Thomson effect. 
Key words: A2. Horizontal ribbon growth, A1. Single crystal growth, A1. Growth models, A1. Interfaces, A1. Interface kinetics, A1. Roughening

* Corresponding author

Email address: peter_kellerman@amat.com

\section{Introduction}

In Horizontal Ribbon Growth (HRG) [1]-[4] heat is removed from the free surface of a molten pool, which causes the top surface to solidify. This solid is then pulled horizontally with a velocity, $u_{s}$, such that a steady-state process is established. We have developed a specific version of HRG called the Floating Silicon Method (FSM) to grow single crystal silicon directly in a form factor suitable for high efficiency solar cells. Since the density of the solid is less that of the liquid, it floats, allowing the sheet to extend over a long length of the melt with no mechanical stress. The novelty of FSM relative to previous HRG techniques is that floating enables the creation of distinct thermal zones having sufficient separation (i.e. much greater than the crucible depth) to allow independent control and optimization of different functions, namely: solidification of the leading edge, thickness control, low-stress separation from the melt, and low-stress removal from the hot furnace (see Figure 1). For example, the ribbon's separation from the melt at the quartz crucible wall occurs in an isothermal region (just above the melt temperature), thus avoiding both thermal stress and issues of freezing to the wall. Also, within the region between the leading edge formation and the separation at the wall, additional heaters provide the heat needed to melt back the ribbon's thickness in a controlled manner, allowing an arbitrarily thin and uniform ribbon to be pulled from the melt. In our test stand, we have been able to produce single- 
crystal (100) ribbons with low dislocations $\left(<1 \mathrm{E} 3 / \mathrm{cm}^{2}\right)$, widths greater than $15 \mathrm{~cm}$, pull speed greater than $2 \mathrm{~mm} / \mathrm{s}$, and thinness below $200 \mu \mathrm{m}$. This can all be done in a continuous manner.

Previous experimental work [3], [4] has provided information such as growth rates, sheet thickness, and types of material grown (single-crystal versus multi-crystal). But heat flow for the different functions was not distinct (i.e. they did not make use of the floating nature of the ribbon), and so they were not able to achieve single crystal, wide, thin, continuous growth. Ciszek [23] observed faceted growth at the surface of a melt, but it was not pulled to form a ribbon.

In the FSM process we have found that a (111) facet forms at the leading edge of the ribbon and that the growth proceeds in a series of abrupt spurts in a saccadic process. A solidification limit cycle theory is proposed to describe this saccadic behavior. The combined facet/roughened interface kinetics of Weinstein and Brandon [5] is generalized to include a kinetic roughening transition, as well as a refaceting mechanism that involves the curvature of the interface. Numeric estimates are included to relate this theory to observations.

\section{Phenomenology}

In FSM, ribbon growth is initiated by inserting a seed cut from a CZ (100) wafer into the furnace, floating it on the melt, and moving it such that its leading edge is within a growth zone. The growth direction is in the [011] direction, although some experiments were performed with seeds cut from a (111) wafer with a [112] growth direction. Stable single crystal growth was achieved by using a narrow line of intense helium cooling above the melt and a heater below the quartz crucible in the growth zone. For example, in order to grow ribbons at a steady state horizontal pull speed of $1 \mathrm{~mm} / \mathrm{s}$, helium cooling with a profile peaked at approximately $100 \mathrm{~W} / \mathrm{cm}^{2}$ and heat flow in the melt of approximately 15 $W / \mathrm{cm}^{2}$ was required. Attempting to pull faster with these conditions resulted in the ribbon pulling out or becoming dendritic. 
This result is quite distinct from the expectation from previous HRG work, where the solidification is described by a "growth wedge" [5], [6]-[13], in which the leading end of the ribbon tapers to zero thickness forming a shallow growth angle. The interface is assumed to be atomically roughened, remaining at the equilibrium melt temperature, with growth velocity determined solely by heat flow, so that one should be able to pull ribbon at arbitrarily fast speeds with a low intensity, broad, heat removal source, with the thickness of the ribbon getting arbitrarily smaller as one pulls faster without limit.

Another feature of ribbons grown with FSM is the presence of "facet lines" on the top surface (Figure 2). For a (100) ribbon, the top surface is generally in the [100] crystal direction, yet is not itself a facet. The top surface is rather the wake of the leading edge as it freezes, and is in a certain sense a "fossil record" of the leading edge freezing process. Ridges or facet lines run laterally across the ribbon, perpendicular to the growth direction, with roughly regular spacing of approximately $10 \mu \mathrm{m}$, ending at the ribbon edges caused by the tapering off of the cooling gas jet towards the sides. The side effects regions are still single crystal, i.e. one finds that there are no grain boundaries or twins. On closer inspection, side effects consist of the mitered joining of angled facet lines. In the case of (100) ribbons pulled in the [110] direction, these mitered joints are at $90^{\circ}$, whereas for (111) ribbons pulled in the [112] direction, the mitered angles are at $120^{\circ}$.

A more detailed study of the top surface morphology was accomplished using a Keyence confocal microscope (Figure 3a). This revealed a surface profile resembling a rounded saw tooth with peak to valley amplitude generally between 0.05 and 0.5 microns; the lines being a visual effect of the saw tooth peaks and valleys. This pattern also exhibits a random element; while the saw teeth generally alternate up and down, at times there may be 2 or more ups or downs in a row. We believe that this is due to the random sampling of the relatively slow melt surface waves by the very fast saccadic facet layering (as will be discussed later in this paper). It should be noted that in solar cell manufacturing, the $\mathrm{KOH}$ 
texturization etch (which is generally > 10um) will eliminate the facet lines, and the side-effects can be melted back while the ribbon is floating in the melt, so that a ribbon will be able to be used as-grown, without additional surface processing steps.

The direction of these facet lines on the ribbon surface can be related to the diamond cubic crystal structure of silicon with space group Fd3m. In the case of a (100) ribbon being grown in the [011] direction, there are 8 possible $\{111\}$ planes that can intersect the surface as shown in Figure $4 a .\{111\}=$

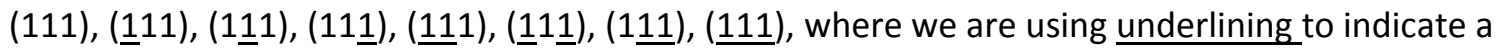
negative Miller index. The intersection of either the (111) or (111) planes with the top surface (100) plane forms a line that is perpendicular to the pull direction, corresponding to the facet lines across the width of the ribbon. If the leading edge of the ribbon were indeed a (111) facet, it would form an "acute" $54.7^{\circ}$ angle with the top surface, while a (111) leading edge would form an "obtuse" $125.3^{\circ}$ angle with the top surface. Meanwhile the intersection of either the (111) or (111) planes with the (100) surface forms a line that is parallel with the pull direction, consistent with the observed $90^{\circ}$ mitered side-effects. These facets also form either an acute $54.7^{\circ}$ or obtuse $125.3^{\circ}$ angle with the top (100) surface, depending on the side of the ribbon one is considering. As there is 4-fold rotational symmetry about the [100] axis, growth in the [011], [011], and [0111] directions will yield identical results as growth in the [011].

In the case of (111) ribbon pulled in the [112] direction, one can visualize the 6 intersecting $\{111\}$ planes as the faces of an octahedron with the (111) and (111) planes parallel to the melt surface, as shown in figure $4 \mathrm{~b}$. The intersection of the (11ㅅ) leading-edge facet plane forms a line that is perpendicular to the pull direction, again corresponding to the facet lines across the width of the ribbon. However, the

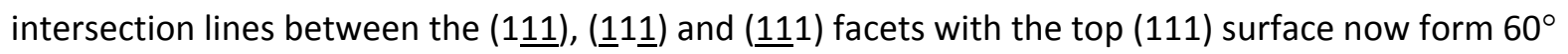
angles with the pull direction, corresponding to the observed $60^{\circ}$ mitered side effects. Note that in the 
case of a (111) ribbon, there is now only 3-fold symmetry, and pulling in the [11] ] direction is not equivalent to pulling in the opposite [112] direction. For the [112] pull direction case, the leading edge (11) facet plane forms an obtuse $109.5^{\circ}$ angle with the top surface (there is no $\{111\}$ plane forming an acute angle with the top (111) surface), while for the [112] pull direction case, the (111) leading edge facet forms $70.5^{\circ}$ acute angle.

The spacing between the facet lines is insensitive to the growth or pull speed of the ribbon (see figure $3 b)$, indicating that the saccadic nature of the facet lines is not the result of sampling some other periodic disturbances in the system such as waves on the surface of the melt or vibration of the equipment. In order to obtain high quality single crystal sheets with high minority carrier lifetime, a quartz crucible is used (as in CZ). But due to the non-wetting nature of molten silicon on quartz, instability arises at the edges of the crucible, creating a wave action on the surface of the melt. This wave action is observed to have a wavelength on the order of $1 \mathrm{~cm}$, an amplitude on the order of $1 \mathrm{um}$, and frequency on the order of $10 \mathrm{~Hz}$. For a ribbon being pulled at $1 \mathrm{~mm} / \mathrm{s}$, the facet line separation of 10um implies a frequency of $100 \mathrm{~Hz}$. Thus, although the surface waves may have an influence on the random aspect of the facet lines, they cannot be the fundamental cause of the facet lines themselves. Also, the He cooling flow rate was varied with no change in facet line spacing, ruling out a gas convective instability as the cause of the saccadic facet lines.

To visualize the shape of the growing front during this saccadic process, we used a high concentration of antimony dissolved in the melt. This method of interface demarcation during crystal growth was developed to study CZ growth [14]-[16]. Because Sb has a low segregation coefficient (0.023) the concentration in the solid is very sensitive to the growth velocity. Regions with slow growth rate result in low $\mathrm{Sb}$ concentration (Sb has time to diffuse away in the melt), while areas of fast growth result in higher Sb concentration. By cross-sectioning a ribbon parallel to the pull direction and performing a 
Wright etch [25], the variation in Sb concentration and variation in growth speed at the ribbon leading edge can be seen. Figure 5 shows a cross section of a (100) ribbon grown from a melt with an Sb atomic fraction of 6.9E-5. The ribbon was etched for 1 minute.

The appearance of lines at angles at $\sim 55^{\circ}$ and $\sim 125^{\circ}$ with the surface confirms not only the existence of $\{111\}$ leading edge facets during growth, but also that both the acute (111) and obtuse (111) facets are both present together and surge synchronously during the saccadic cycles. The depth of the intersection line between the two facets can vary, at times being very shallow so that only the acute facet is apparent, and we observe the lower acute facet continuing to a depth of $\sim 100 \mu m$, at which point the $\mathrm{Sb}$ demarcation becomes unclear. That is, that the saccadic nature of the leading edge growth prevails under the surface of the melt through a significant depth. The saccadic nature of the growth appears to be intrinsic to the leading edge FSM solidification process.

\section{Solidification Limit Cycle Theory}

In this section, we present a continuum model of solidification that is consistent with the observed phenomenology. In our model, the solidification process is described by: 1- heat flow in the melt and solid as governed by the thermal field equations, 2- latent heat released at the interface depending on growth rate, as expressed by a discontinuity of heat flux at the interface, and 3-attachment kinetics at the interface. The attachment kinetic expression relates the growth speed at that point to the temperature and geometry of the interface. Together, these relationships completely determine the thermal field and interface growth speed. The undercooling of the solid-liquid interface can also be considered as the driving force in this non-equilibrium thermodynamic process.

The kinetic expression is of the form $v_{g}=\beta \Delta T$, where $v_{g}$ is the growth rate of the solid-liquid interface, $\Delta T$ is the undercooling of the interface, and $\beta$ is the attachment coefficient. For atomically roughened 
growth, the attachment coefficient $\beta=\beta_{\text {rough }}$ is independent of temperature and interface orientation, and has a high value of approximately $12 \mathrm{~mm} /(s \mathrm{~K})$ [7], [17], so that the interface remains close to the equilibrium melt temperature for a growth speed of $1-2 \mathrm{~mm} / \mathrm{s}$. Also, since the kinetics are isotropic, the shape of the interface follows the $T_{m}$ isotherm in the liquid. This isotropic roughened continuum model was the basis of previous discussions of HRG.

In order to describe the observed faceted growth, $\beta$ must be highly anisotropic, dropping to a low value when the interface is aligned with a $\{111\}$ crystallographic plane. Also, when driving facet growth at large undercooling, $\beta$ increases nonlinearly, eventually reaching a kinetic roughing point where the interface suddenly loses its crystallographic facet and becomes atomically roughened. At these large values of $\Delta T$ the model becomes isotropic. The functional dependence of $\beta$ on $\Delta T$ and $\theta$ have been related to interface growth mechanisms, such as "growth by two-dimensional nucleation (2DN)", and "step-flow mechanism (SM)".

$$
\begin{array}{cl}
\beta_{S M}(\theta) & =B_{s t}|\sin \theta| \\
\beta_{2 D M}(\theta, \Delta T) & =\cos \theta \cdot B e^{-A / \Delta T,}
\end{array}
$$

where the constants are $B_{s t} \sim 63 \mathrm{~cm} / \mathrm{sK}, B \sim 1.5 \times 10^{12} \mathrm{~cm} / \mathrm{sK}$, and $A \sim 140 \mathrm{~K}$ [5], [17], [18]. These dependencies have been combined into a single smooth function $\beta(\theta, \Delta T)$ by Weinstein and Brandon [5]. This expression, as depicted in Figure 6b, along with the thermal field equations, was used to develop an FEM model of combined roughened and faceted growth in a Vertical Gradient Freeze furnace, as well as CZ growth. Note that this functional assemblage extends beyond the experimentally verified range. For large $\Delta T, \beta$ is shown to increase exponentially (in the $\theta=0\{111\}$ plane), smoothly connecting to the roughened value (as in Equation 1). But this implies a facet growth rate of several $\mathrm{cm} / \mathrm{s}$ ! It is well known that at some $\Delta T^{*}$ there is "kinetic roughening" transition, and the interface defacets [7]. 
Helenbrook $[19,20]$ has created a continuum FEM solidification model using the Weinstein and Brandon $\beta(\theta, \Delta T)$ that describes steady-state faceted edge growth for an acute (111) facet leading edge on a (100) ribbon (Figure 6a). The cooling profile on the surface was calculated separately, with the simplification that the surface was isothermal at the melt temperature $T_{m}$. The discontinuity in cooling shown is due to the difference in emissivity between the solid and liquid. The solid is being pulled to the right, while the solid growing to the left, with the melt flowing into the solid-liquid interface such that the leading edge remains stationary and the condition $v_{g}=v_{p u l l} \sin \left(\theta-54.7^{\circ}\right)$ is met at all points. This occurs by trading off interface undercooling $\Delta T$ with misorientation angle $\theta$ on the $\beta(\theta, \Delta T)$ curve, as shown in Figure 6a as the locus of points on the beta surface corresponding to the leading edge interface. An important result of this model is that in order to obtain the observed high pull speeds, the melt must be supercooled in front of the facet portion of the interface, and as the facet transitions to roughened growth at the "elbow" of the leading edge, the liquid temperature becomes higher than $T_{m}$, thereby providing stability. The supercooling of the melt in front of the faceted leading edge was also recently described by a heuristic scaling theory of FSM [24]. This balance between intense cooling at the leading edge from above the melt while having intense heating under the leading edge is enabled by the FSM architecture. This model accurately matches many of the experimental phenomena, such as the precise location of the leading edge in the profile of the helium cooling jet and how it changes with pull speed and other experimental factors, and is the subject of a companion paper [20]. According to this model, one should be able to drive steady-state faceted edge growth to high speeds ( $>5 \mathrm{~mm} / \mathrm{s}$ ), provided that sufficiently intense heat removal is provided.

A problem with this model is that it does not predict any sort of saccadic phenomenon, and so does not explain the observed facet lines. We believe that the kinetics needs to be extended to include other physical phenomena, and propose a heuristic Limit Cycle Theory, which explains the observed saccadic growth as shown in Figure 7. Figure 7a shows the thermal field of a dual facet leading edge. The 
isotherms have been estimated based on the steady-state single facet FEM model of Helenbrook [20]. Because the facet growth is inhibited, the facet interface becomes supercooled, and a supercooled pool forms in the melt in front of the leading edge. Since the bottom of the ribbon is roughened growth, its interface temperature remains close to $T_{m}$, and the extent of the supercooled pool is confined to roughly the leading edge facet length (on the order of $100 \mu \mathrm{m}$ ). Since heat flows from the interface into the supercooled melt as well as into the solid (stability being provided by the facets), the coldest point is below the melt surface, and is depicted here at the facet intersection point.

At some critical undercooling of $\Delta \mathrm{T}^{*} \sim 5 \mathrm{~K}$ [7], growth at that point is then un-inhibited, and the attachment coefficient goes to its high roughened value; growth surges, releasing latent heat, causing the interface temperature to rise near $T_{m}$. This morphologically unstable roughened growth will surge into the supercooled pool in the form of a protuberance (Figure 7b). Although the tip of this protuberance includes small areas aligned with the two $\{111\}$ planes that have a tendency to form facets, we suggest that it does not re-facet until the tip radius increases beyond some critical value $R^{*}$. This decrease in curvature occurs as the surge locally heats the supercooled pool, lengthens, slows and thickens. Once the re-faceting occurs (Figure 7c), the roughened growth will be confined within these facet planes, and the facet layer will fill in, both vertically (Figure 7d), as well as horizontally across the ribbon interface (Figure 8) in both directions across the entire width of the growing ribbon, which in our experiments is more than $15 \mathrm{~cm}$ across. It even progresses similarly around the mitered corners at the edges of the ribbon, as seen in Figure 2. The cycle then repeats. The presence of any dislocations crossing the surface of the supercooled facet would cause continuous nucleation and steady-state growth, so we interpret our facet line data to indicate dislocation free crystal. Dislocations seen in our ribbons must thus be due to subsequent stresses.

This type of unstable crystal growth surge into a supercooled melt has been studied in the context of dendritic growth of metals. Ivantsov [21] solved the thermal field equations for steady-state growth of a parabolic needle, and found that the product of the growth velocity $v_{\text {tip }}$ and the radius of curvature $R_{\text {tip }}$ at the paraboloid tip is related to the undercooling of the pool (far from the tip). 


$$
\begin{gathered}
\frac{v_{t i p} \cdot R_{t i p}}{2 \alpha} \equiv P e=\text { function of }(\Delta \vartheta) \quad \text { Equation2 } \\
\Delta \vartheta \equiv \frac{\Delta T}{\Delta H_{f} / C_{p}} \text { is the dimensionless temperature } \\
\frac{v_{t i p} \cdot R_{t i p}}{2 \alpha}=P e=.647 \cdot \Delta \vartheta^{1.27} \quad(.001<\Delta \vartheta<.05)
\end{gathered}
$$

Horvay and Cahn [22] generalized this result for a parabolic platelet, finding a similar result, although with slower penetration velocity into the melt, due to the two-dimensional nature of the platelet as compared with the parabolic needle. In the case of faceted edge growth Limit Cycle surge, the situation is somewhere between the two cases.

Equation 2 only defines the product of $\left(v_{\text {tip }} \cdot R_{\text {tip }}\right)$ as a function of temperature; i.e. the parabolic needle could grow infinitely fast while being infinitely narrow. It is well known, however, that surface energy effects limit the curvature of a solidification interface. The Gibbs Thomson Effect relates the curvature of the interface with a decrease in the interface temperature below that of the (flat) equilibrium melt temperature $T_{m}$.

$$
T_{e q}=T_{m}-\frac{2 \gamma_{s l} T_{m}}{\rho \Delta H_{f}}\left(\frac{1}{R}\right) \quad \text { Equation } 3
$$

In the case of a parabolic needle, this has a stabilizing effect. If the tip radius becomes too small, the interface temperature drops, limiting the release of latent heat into the subcooled liquid and slowing the growth and increasing the tip radius in accordance with Equation 2. The smallest possible tip radius occurs when the tip interface temperature is equal to the surrounding liquid temperature, which can used to obtain the limit of surge tip radius and velocity:

$$
R_{\text {tip }}>\frac{2 \cdot \gamma_{s l} \cdot T_{m}}{\rho \cdot H_{f}}\left(\frac{1}{\Delta T}\right), \quad \text { Equation } 4
$$


where $\Delta T$ is the undercooling of the liquid near the tip. For an initial $\Delta T \sim 5 K, R_{t i p}>.02 u m$, and $v_{\text {tip }}<100 \mathrm{~cm} / \mathrm{s}$.

In Ivantsov's analysis no faceting effects were considered although the rounded tip of this unstable surge growth must sample the $\{111\}$ planes. In order for this roughened surge to occur, the facet inhibition must "turn off", showing a high kinetic attachment coefficient $B$, which according to the standard model is a function of only the undercooling and misorientation angle. One way to accommodate this in a continuum model, is to add a dependence on curvature, such that if a point on the interface which is sampling the facet plane has a radius of curvature below some critical value, it will remain roughened (beta very large) and will not facet (beta very low). In this heuristic extension of the existing models, the attachment coefficient is dependent on three parameters. $\beta(\Delta T, \theta, R)$ depends on the supercooling, the crystallographic angle of the surface and the local radius of curvature.

The degree of misorientation from the $\{111\}$ plane needed to obtain atomic roughening attachment is generally estimated to be $\sim 1^{\circ}$ [7]. For high curvature, this limits the extent of interface surface that can nucleate to form a facet. For a radius of curvature of $0.5 \mathrm{um}$, an arc-length of $1^{\circ}$ corresponds to only $\sim 100 \AA$, or only $\sim 15$ lattice spacings for Si. On an atomic level, this amounts to a requirement that a facet grows to a critical size before it can effectively reduce the attachment coefficient. In a thermodynamic perspective, thermal fluctuations of such a small interface would exceed the $1^{\circ}$ misorientation limit, preventing it from being a viable facet "nucleation" site.

As the surge advances, the temperature of the pool increases due to release of latent heat, the radius of the tip increase, and the rate of growth decreases. By the time the melt has warmed to $\sim 1 K$ below $T_{m}$, the tip radius has increased to $\sim 1 \mathrm{um}$, the growth speed has slowed to $<1 \mathrm{~cm} / \mathrm{s}$, in time $d t \sim 10 \mathrm{um} / 50 \mathrm{~cm} / \mathrm{s}=20 \mathrm{us}$. The length of this surge growth can be estimated (using the area of a parabola) as 


$$
R_{\text {pool }} \sim \sqrt{\frac{2 R_{t i p^{\frac{1}{2}} \cdot L_{\text {surge }}{ }^{\frac{3}{2}} H_{f} \rho}}{C_{p} \rho \cdot \Delta T}}
$$

Equation 5

So for a surge length of $\sim 10 \mathrm{um}$ (the observed spacing between facet lines), the size of the pool $R_{\text {pool }}$, which is commensurate with the size of the leading edge facet, is $\sim 0.125 \mathrm{~mm}$, in rough agreement with the Sb demarcation observations.

Once re-faceting occurs the speed of this "facet layer flow", $v_{l}$ can be estimated by considering the latent heat generated by this growing roughened layer edge, and the rate at which this heat can be dissipated into the solid through a boundary layer of width $\mathrm{h}$ (see Figure 8).

The heat generated (per unit length of the facet), $P$, can be related to the thickness of the layer, $t$, by $P \sim \rho L_{f} \cdot t \cdot v_{l}$, where $\rho$ is the density and $L_{f}$ is the latent heat of fusion. This generated heat is locally conducted through the boundary layer, yielding

$$
\frac{k \cdot \Delta T}{h} \sim \frac{\rho \cdot L_{f} \cdot t \cdot v_{l}}{h}
$$

where $k$ is the thermal conductivity. So that

$$
v_{l} \sim \frac{k \Delta T}{\rho L_{f} t}
$$

Equation 6

From the steady-state FEM model, we see that the solid can be significantly supercooled under the He jet (on the order of $\Delta T_{\text {solid }} \sim 100 \mathrm{~K}$ ). With a layer thickness of $\sim 10 \mathrm{um}$ (observed facet line separation), this yields a facet flow velocity of $\sim 5 \mathrm{~cm} / \mathrm{s}$.

From these estimates, we see that there are very different scales of growth speed. The Ivantsov surge speed is $\sim 50 \mathrm{~cm} / \mathrm{s}$, the surge occurring over a time period of $\sim 20 \mathrm{us}$. The facet layer flows at a speed of 
$\sim 5 \mathrm{~cm} / \mathrm{s}$, taking $\sim 1 \mathrm{~ms}$ to form a $50 \mathrm{um}$ deep leading edge facet. Meanwhile, the average growth speed in agreement with the steady-state model is $\sim 1 \mathrm{~mm} / \mathrm{s}$, so that there is $\sim 10 \mathrm{~ms}$ between surges.

In order to create a more detailed FEM model that includes the limit cycle as described above, the facet kinetics would have to be generalized to explicitly include kinetic roughening (onset of the surge), and curvature dependence (re-faceting). The original 2D nucleation expression (equation 1) predicts facet growth with beta extending all the way up to the roughened growth value, i.e. uninhibited facet growth as fast as roughened growth. Such steady-state facet growth is stable, in the sense that a positive slope implies that as $\beta$ increases (thereby increasing growth rate) the interface temperature lowers, thereby inhibiting heat removal and growth. We suggest that in order to obtain Ivantsov surge behavior, there needs to be an additional kinetic roughening branch that is unstable (negative slope), causing the interface to rapidly warm near $\mathrm{T}_{\mathrm{m}}$, which in turn causes the rapid heat loss to the cold environment. This unstable kinetic roughening branch would occur in this new curvature dimension.

\section{Conclusions}

In this paper, we have described FSM Si ribbon growth, a new method of single-crystal ribbon growth, capable of producing high efficiency, low cost solar wafers. Our observation of both acute and obtuse $\{111\}$ regularly spaced facets across wide ribbons in a range of conditions and Sb demarcation experiments suggest strongly that the leading edge facet grows saccadically, alternating between slow facet growth and fast roughened growth. We have proposed a solidification limit cycle theory to explain this saccadic facet growth. This theory requires generalizing the combined facet-roughened kinetics of Weinstein and Brandon such that the attachment coefficient explicitly contains a kinetic roughening transition, allowing a high-curvature unstable surge in growth. We also propose that this surge growth can only re-facet when the curvature drops below a critical value. Although our description of this theory is heuristic in nature, we have provided estimates of quantities which conform well with our 
observations. More work is needed to incorporate such generalized kinetics directly in a solidification FEM model, which would then provide a more predictive formalism to relate to experiments, as well as provide the foundation for further optimization of the FSM commercial production of single crystal Si ribbon for solar.

\section{Acknowledgements}

Thanks to members of the FSM engineering team for their contributions to the experimental aspects of this work, especially Dave Morrell, Nandish Desai, Chuck Bowen, and Ala Moradian. Thanks as well to the many fruitful discussions with Marty Glicksman, Tom Surek, and Jeff Derby. Early parts of this research were supported, in part, by the US Department of Energy [grant number DE-EE0000595].

\section{References}

[1] William Shockley, Process for Growing Single Crystals. April 1962. US Patent 3,031,275 A, http://www.google.com/patents/US3031275

[2] C. Bleil, “A New Method for Growing Crystal Ribbons," J. Cryst. Growth, vol. 5, no. 2, pp. 99-104, 1969.

[3] H. Bates and D. Jewett, "Low Angle Silicon Sheet Growth: A Review of Progress, Problems and Promise," in Flat-Plate Solar Array Proj. Res. Forum on the High-Speed Growth and Characterization of Crystals for Solar Cells, 1984, vol. 1, pp. 297-307.

[4] B. Kudo, "Improvements in the Horizontal Ribbon Growth Technique for Single Crystal Silicon," J. Cryst. Growth, vol. 50, no. 1, pp. 247-259, 1980.

[5] O. Weinstein and S. Brandon, "Dynamics of partially faceted melt/crystal interfaces I: computational approach and single step-source calculations," J. Cryst. Growth, vol. 268, no. 1, pp. 299-319, 2004.

[6] G. Hoyler, R. Falckenberg, J. Grabmaier, and B. Freienstein, "Fast Si Sheet Growth by the Horizontal Supported Web Technique," J. Cryst. Growth, vol. 79, no. 1-3, pp. 572-577, 1986. 
[7] C. E. Bleil, "A New Approach to Continuous Crystal Sheet Growth," J. Cryst. Growth, vol. 104, no. 1, pp. 29-33, 1990.

[8] J. A. Zoutendyk, "Analysis of Forced Convection Heat Flow Effects in Horizontal Ribbon Growth from the Melt," J. Cryst. Growth, vol. 50, no. 1, pp. 83-93, 1980.

[9] J. A. Zoutendyk, "Theoretical Analysis of Heat Flow in Horizontal Ribbon Growth from a Melt," J. Appl. Phys., vol. 49, no. 7, pp. 3927-3932, 1978.

[10] M. Glicksman and P. Voorhees, “Analysis of Morphologically Stable Horizontal Ribbon Crystal Growth," J. Electron. Mater., vol. 12, no. 1, pp. 161-179, 1983.

[11] P. Daggolu, A. Yeckel, C. E. Bleil, and J. J. Derby, "Thermal-Capillary Analysis of the Horizontal Ribbon Growth of Silicon Crystals," J. Cryst. Growth, vol. 355, no. 1, pp. 129-139, 2012.

[12] P. D. Thomas and R. A. Brown, "Rate Limits in Silicon Sheet Growth: The Connections between Vertical and Horizontal Methods," J. Cryst. Growth, vol. 82, no. 1, pp. 1-9, 1987.

[13]S. Ranjan, S. Balaji, R. A. Panella, and B. E. Ydstie, "Silicon Solar Cell Production," Comput. Chem. Eng., vol. 35, pp. 1439-1453, 2011.

[14] A. Murgai, H. C. Gatos, and A. F. Witt, "Quantitative Analysis of Microsegregation in Silicon Grown by the Czochralski Method," J. Electrochem. Soc., vol. 123, no. 2, pp. 224-229, 1976.

[15]A. Murgai, H. C. Gatos, and W. A. Westdorp, "Effect of Microscopic Growth Rate on Oxygen Microsegregation and Swirl Defect Distribution in Czochralski-Grown Silicon.," J. Electrochem. Soc., vol. 126, no. 12, pp. $2240-2245,1979$.

[16]A. F. Witt, M. Lichtensteiger, and H. C. Gatos, "Application of Interface Demarcation to the Study of Facet Growth and Segregation: Germanium," J Electrochem Soc USA, vol. 121, no. 6, pp. 787 - 90, 1974.

[17] V. V. Voronkov and V. M. Pankov, "The growth of silicon crystals with single dislocations," Kristallografiya, vol. 20, pp. 1145-1151, 1975. 
[18] K. M. Beatty and K. A. Jackson, "Monte Carlo Modeling of Silicon Crystal Growth," J. Cryst. Growth, vol. 211 , no. 1 , pp. 13-17, 2000.

[19] B. T. Helenbrook, "High-order adaptive arbitrary-Lagrangian-Eulerian (ALE) simulations of solidification," Int J Num Meth Eng, vol. in revision, 2016.

[20] B. T. Helenbrook, P. Kellerman, Carlson, F. M., N. Desai, and D. Sun, "Experimental and Numerical Investigation of the Horizontal Ribbon Growth Process," J Cryst. Growth, vol. submitted, 2016.

[21] M. E. Glicksman, Principles of Solidification. Springer Science+ Business Media, 2011.

[22] G. Horvay and J. Cahn, "Dendritic and spheroidal growth," Acta Metall., vol. 9, no. 7, pp. 695-705, 1961.

[23] T.F.Ciszek, "Solid/Melt Interface Studies of High Speed Silicon Sheet Growth", SERI/PR-212-2634, 1985

[24] A.S. Greenlee, Ph.D. thesis, MIT, 2015

[25] Margaret Wright Jenkins, Journal of the Electrochemical Society, vol. 124, pp. 757-759, (1977)

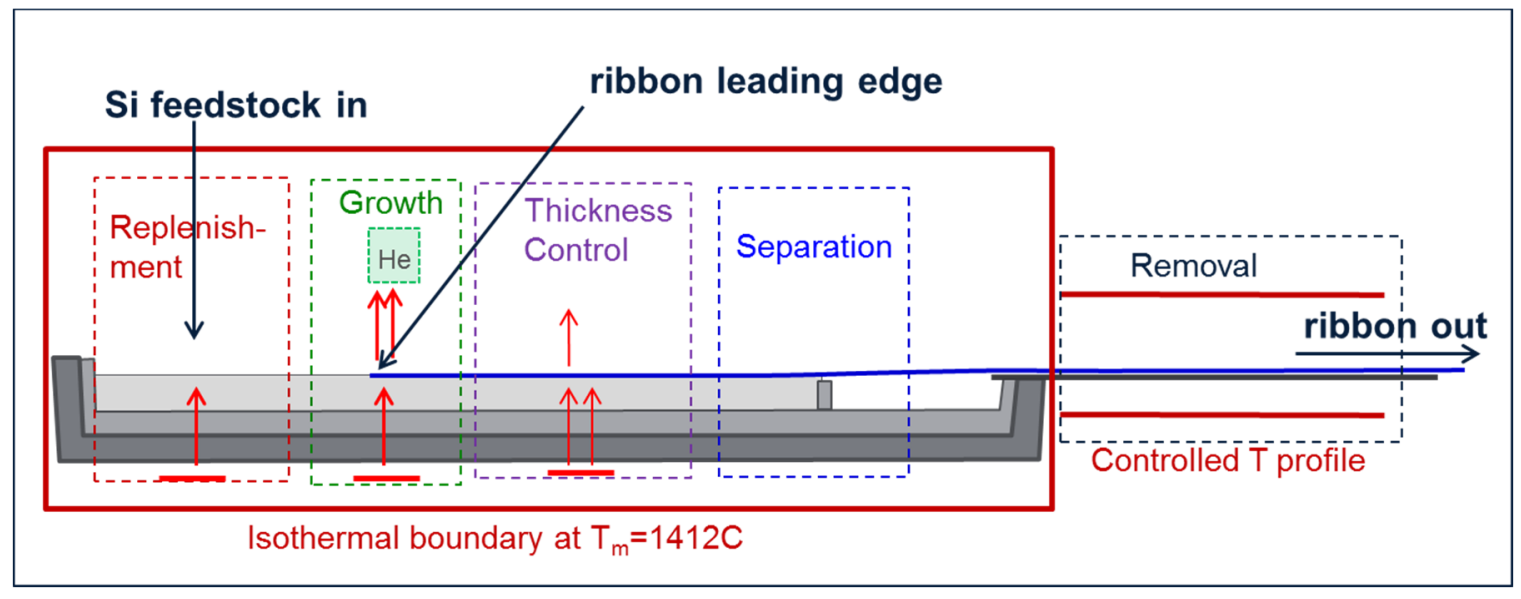

Figure 1: Schematic depiction of the continuous FSM process. The floating ribbon enables localized heat flow zones optimized for the different functions of crystal growth. 


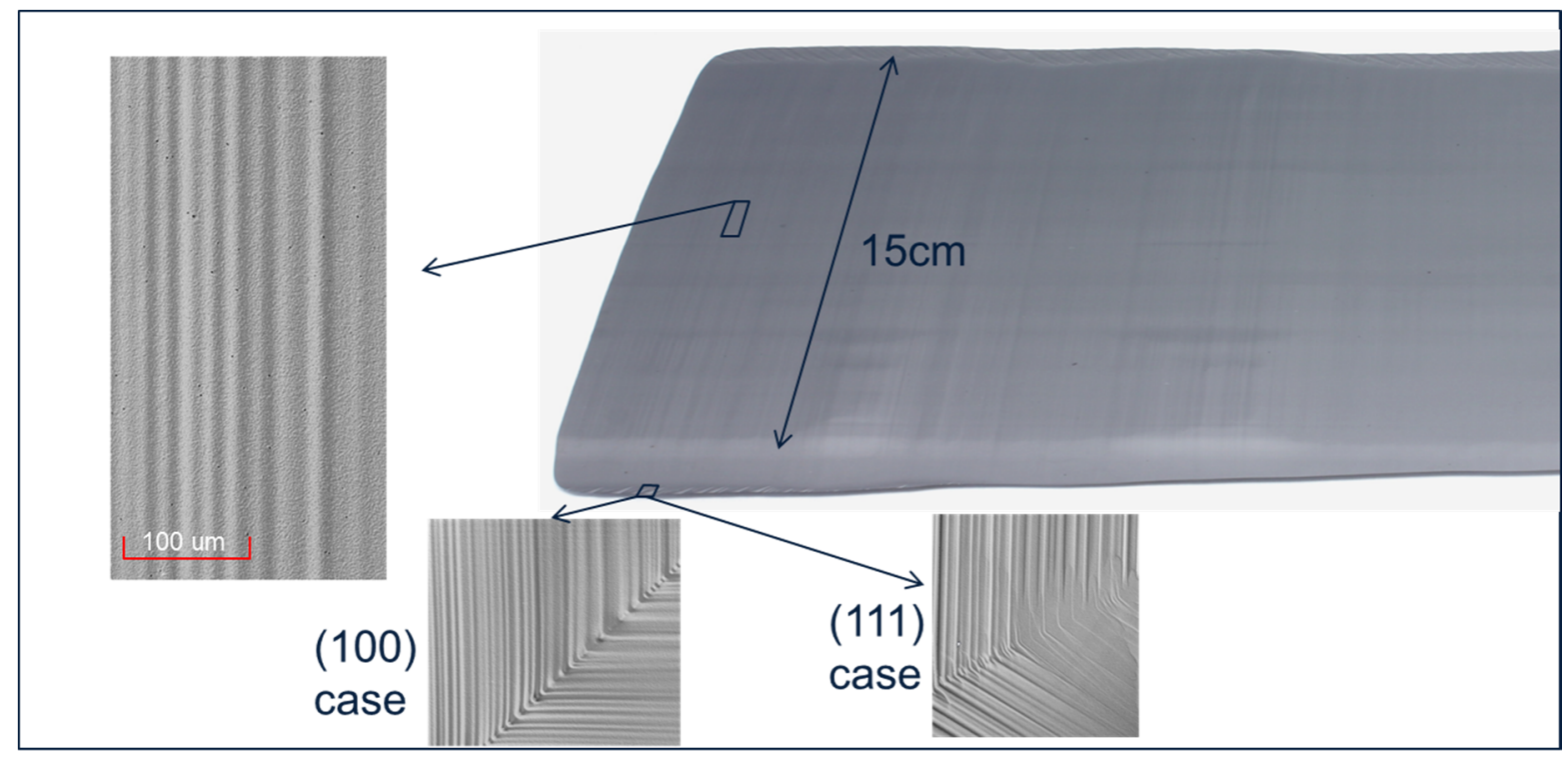

Figure 2: Picture of typical top surface of FSM ribbons, with micrographs of the transverse facet lines and "side effects" for both (100) and (111) cases. 


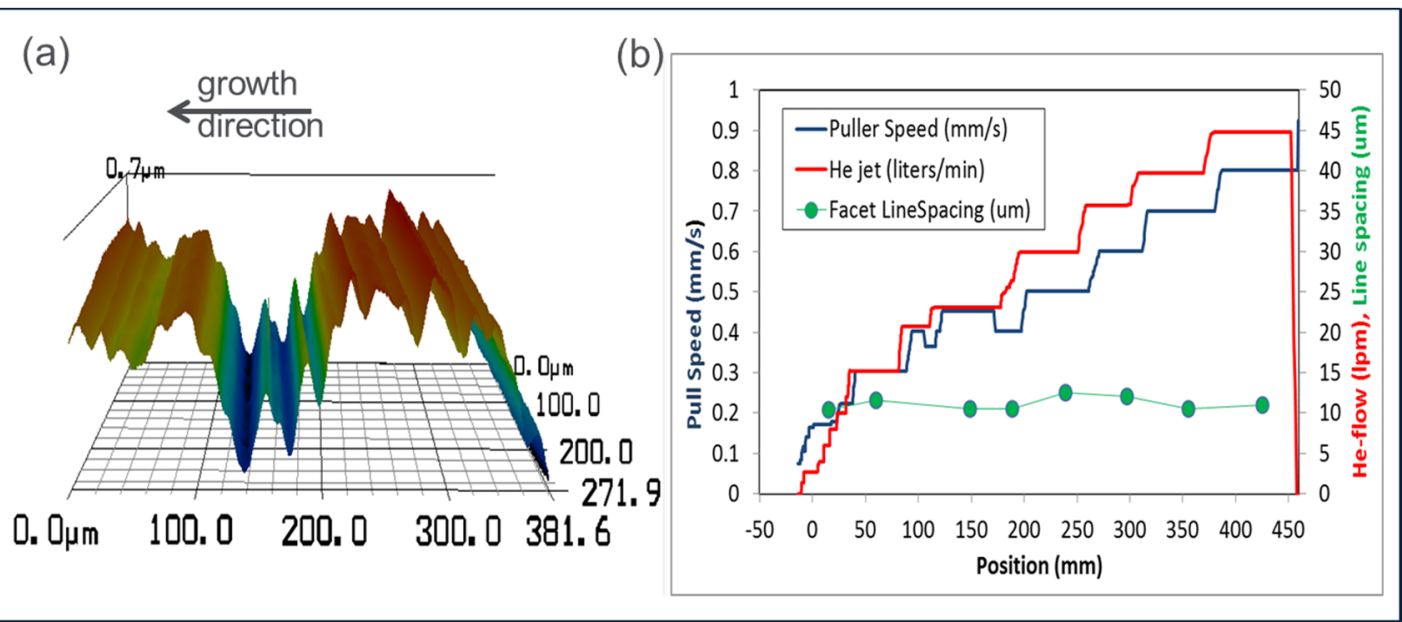

Figure 3: (a) Keyence confocal microscope data showing typical transverse facet lines on the top surface of FSM ribbons. (b) FSM run-time data showing pull speed and He cooling as a function of calculated location on the ribbon. The "peak to valley" line spacing measured using the Keyence was seen to be insensitive to pull speed.

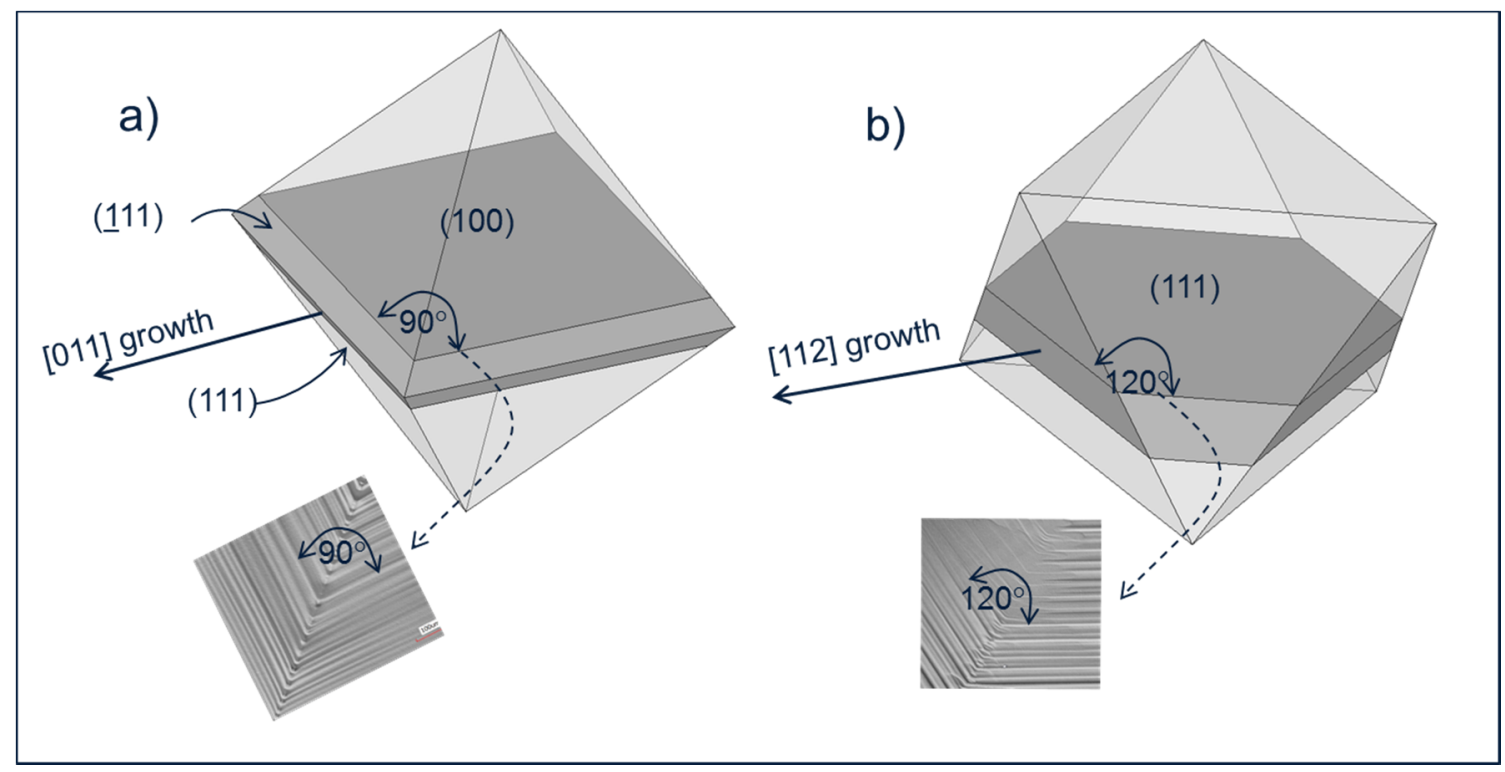

Figure 4: a) Four-fold symmetry of (100) ribbon grown in [011] direction; b) Three-fold symmetry of (111) ribbon. Corresponding "side-effect" micrographs are also shown. 


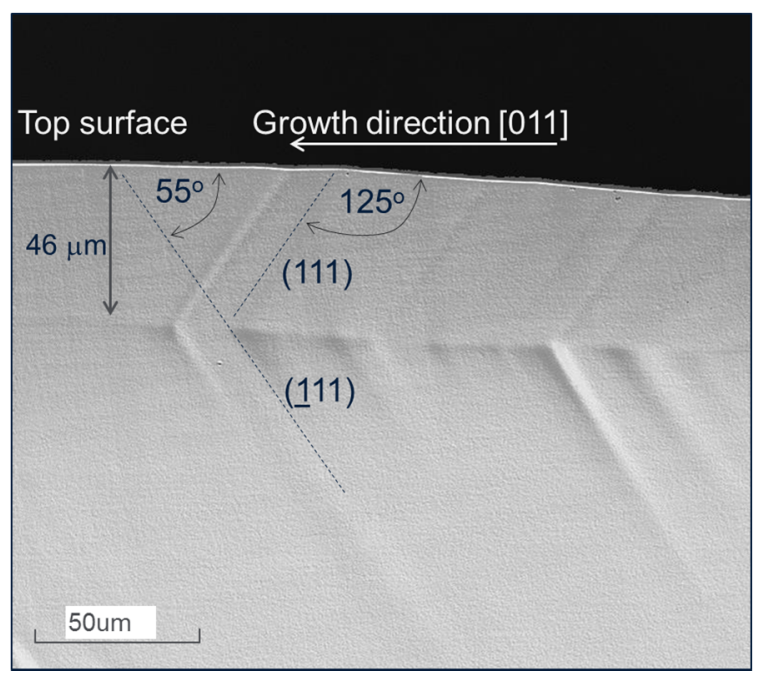

Figure 5: Antimony demarcation image, showing dual $\{111\}$ facets. Varying concentrations of Sb (light and dark) indicate sharply varying growth speeds.

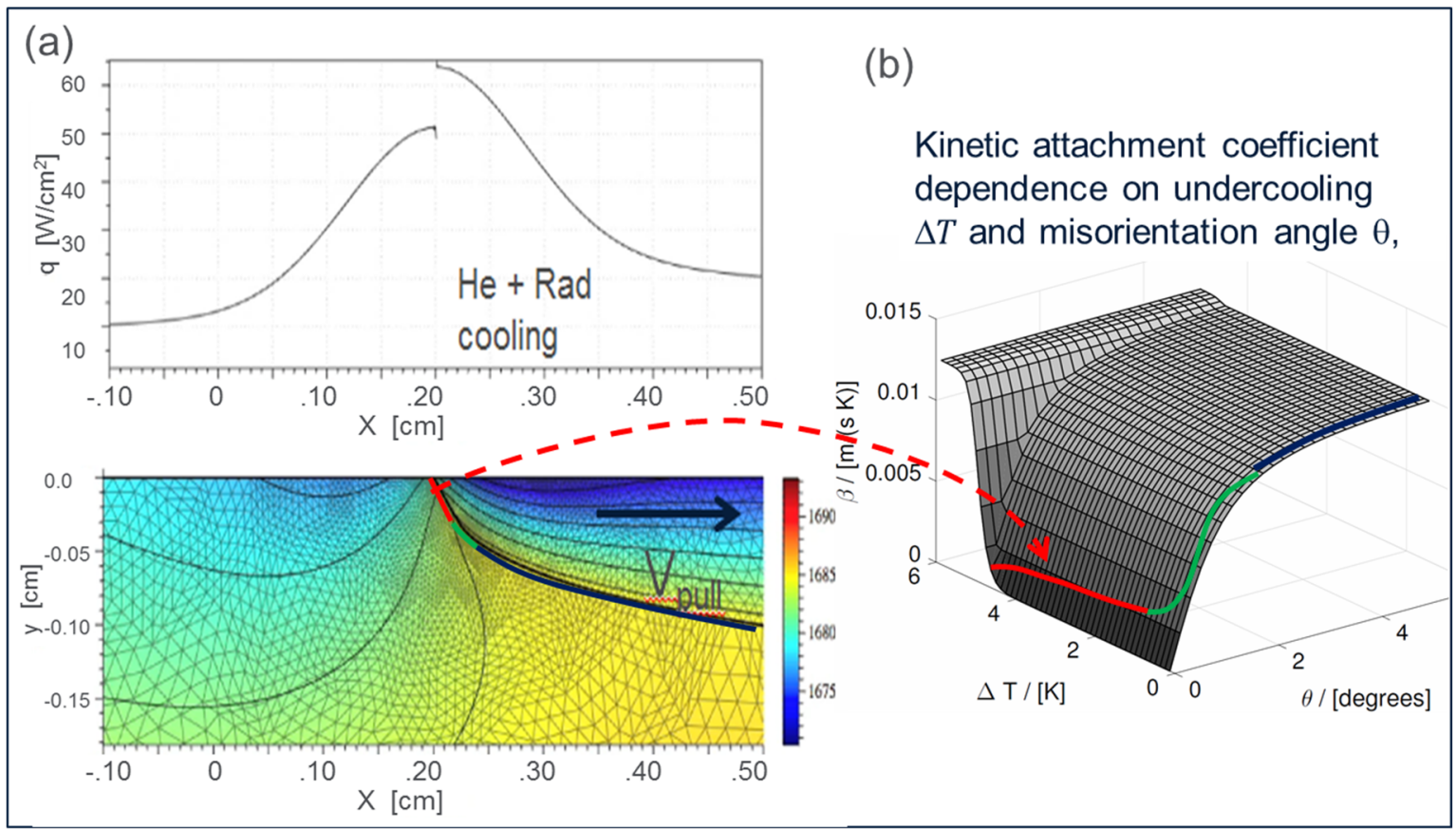

Figure 6: (a) Steady-state faceted edge growth model of Helenbrook, showing the leading edge facet (red), transition (green), and roughened growth segments (black) as a locus of points on the kinetic attachment $\beta$ curve (b) of Weinstein and Brandon [5]. Isotherms shown are $2 \mathrm{~K}$ apart. 


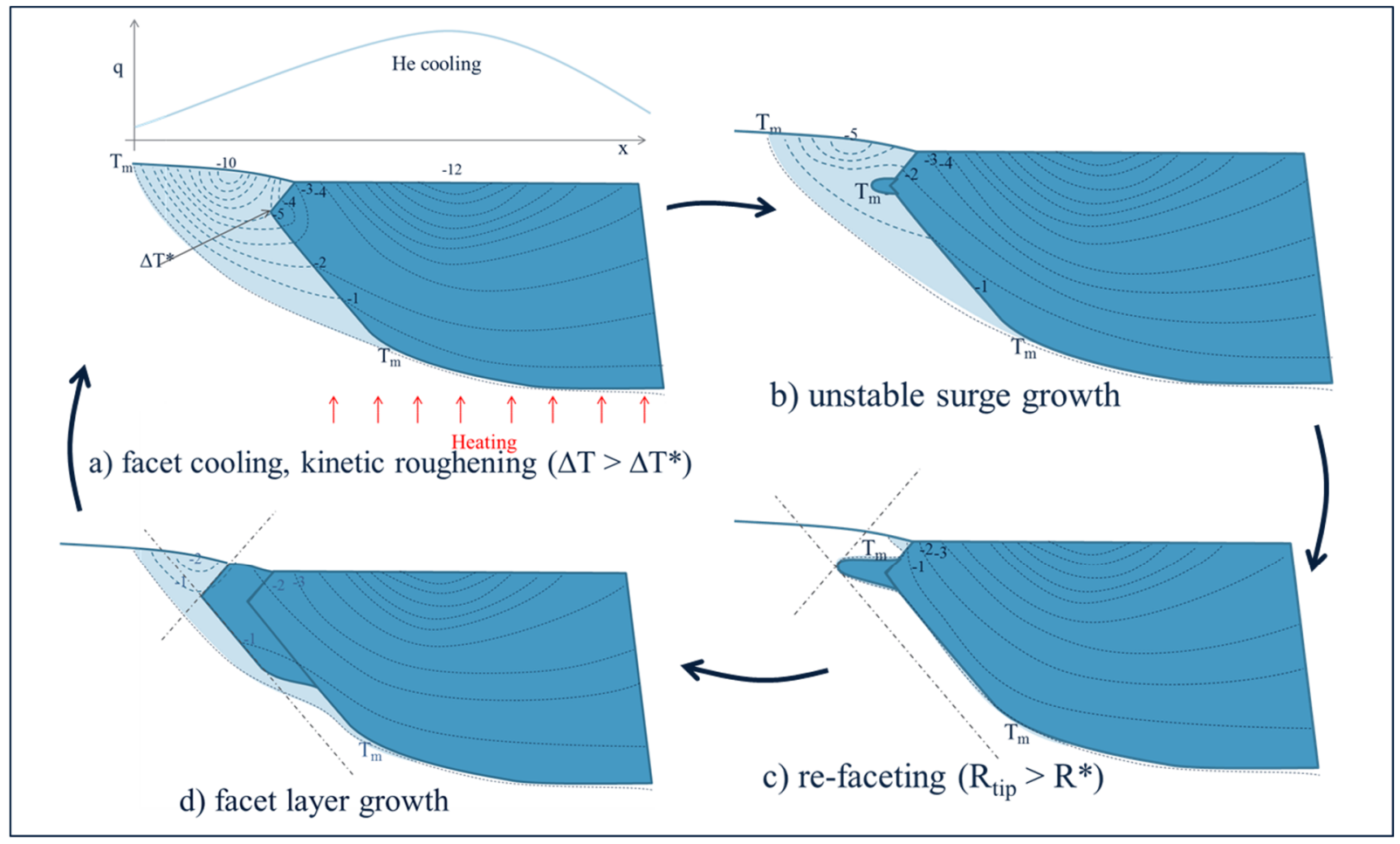

Figure7: Faceted edge growth solidification limit cycle: (a) dual facet chills, kinetic roughening temperature $T^{*}$ at some point on interface; (b) unstable protuberance surges into super-cooled pool; (c) surge slows, tip radius increases above critical value $R^{*}$ and tip re-facets; $d$ - facet layer grows across entire leading edge. Repeat.

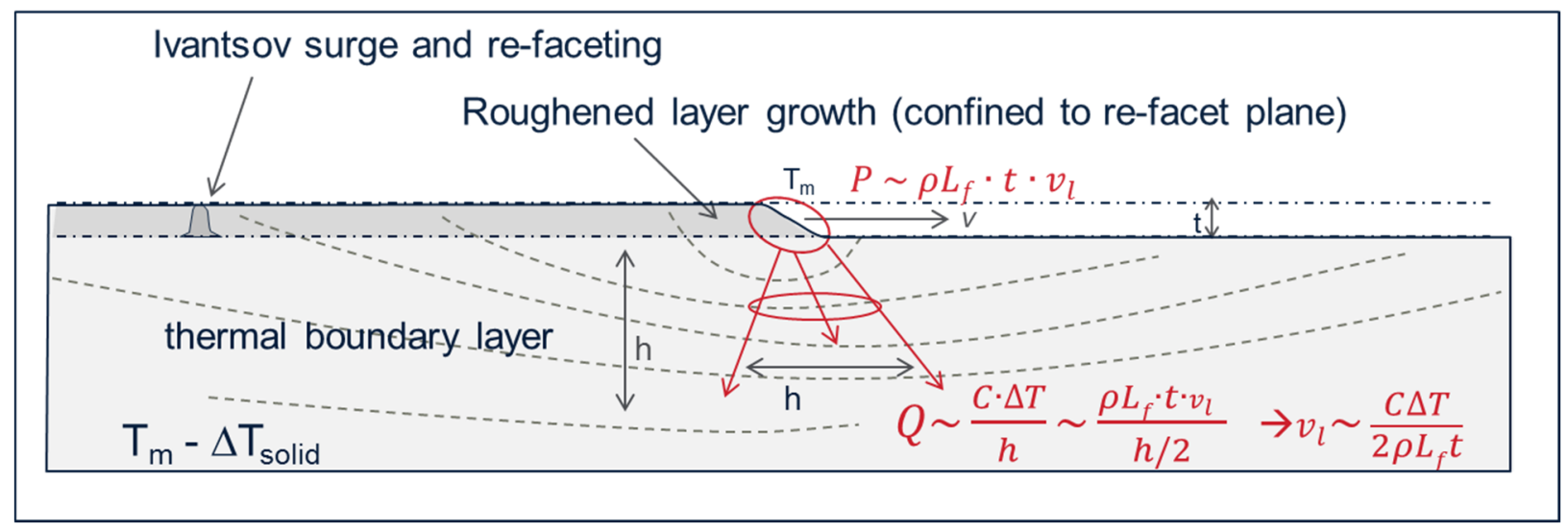

Figure 8: Depiction of facet layer growth with the latent heat generated at the growing edge of the layer being conductively dissipated to the supercooled solid through a boundary layer. 